Corresponding author: tamim. shaikh@ucdenver.edu

(c) 2016 Yu et al. This article is distributed under the terms of the Creative Commons Attribution-NonCommercial License, which permits reuse and redistribution, except for commercial purposes, provided that the original author and source are credited.

Ontology terms: intellectual disability, mild; microcephaly; restrictive cardiomyopathy; severe short stature

Published by Cold Spring Harbor Laboratory Press

doi: $10.1101 / \mathrm{mcs} . a 000844$

\section{Discovery of a potentially deleterious variant in TMEM87B in a patient with a hemizygous $2 q 13$ microdeletion suggests a recessive condition characterized by congenital heart disease and restrictive cardiomyopathy}

\author{
Hung-Chun Yu, ${ }^{1,4}$ Curtis R. Coughlin, ${ }^{1,4}$ Elizabeth A. Geiger, ${ }^{1}$ Blake J. Salvador, ${ }^{1}$ \\ Ellen R. Elias, ${ }^{1}$ Jean L. Cavanaugh, ${ }^{2}$ Kathryn C. Chatfield, ${ }^{2}$ Shelley D. Miyamoto, ${ }^{2}$ \\ and Tamim H. Shaikh ${ }^{1,3}$ \\ ${ }^{1}$ Department of Pediatrics, Section of Genetics, University of Colorado School of Medicine, Aurora, Colorado \\ 80045, USA; ${ }^{2}$ Department of Pediatrics, Division of Cardiology, University of Colorado School of Medicine, \\ Aurora, Colorado 80045, USA; ${ }^{3}$ Colorado Intellectual and Developmental Disabilities Research Center \\ (IDDRC), University of Colorado School of Medicine, Aurora, Colorado 80045, USA
}

[Supplemental material is available for this article.]

\begin{abstract}
Restrictive cardiomyopathy $(\mathrm{RCM})$ is a rare cause of heart muscle disease with the highest mortality rate among cardiomyopathy types. The etiology of RCM is poorly understood, although genetic causes have been implicated, and syndromic associations have been described. Here, we describe a patient with an atrial septal defect and restrictive cardiomyopathy along with craniofacial anomalies and intellectual disabilities. Initial screening using chromosomal microarray analysis (CMA) identified a maternally inherited $2 q 13$ microdeletion. The patient had many of the features reported in previous cases with the recurrent $2 q_{13}$ microdeletion syndrome. However, the inheritance of the microdeletion from an unaffected mother combined with the low incidence (10\%) and milder forms of cardiac defects in previously reported cases made the clinical significance of the CMA results unclear. Whole-exome sequencing (WES) with triobased analysis was performed and identified a paternally inherited TMEM87B mutation (c.1366A>G, p.Asn456Asp) in the patient. TMEM87B, a highly conserved, transmembrane protein of currently unknown function, lies within the critical region of the recurrent $2 q 13$ microdeletion syndrome. Furthermore, a recent study had demonstrated that depletion of TMEM87B in zebrafish embryos affected cardiac development and led to cardiac hypoplasia. Thus, by combining CMA and WES, we potentially uncover an autosomal-recessive disorder characterized by a severe cardiac phenotype caused by mutations in TMEM87B. This study expands the spectrum of phenotypes associated with the recurrent $2 q 13$ microdeletion syndrome and also further suggests the role of TMEM87B in its etiology, especially the cardiac pathology.
\end{abstract}




\section{INTRODUCTION}

The incidence of cardiomyopathy in children is only 1-2 per 100,000 per year, but it is the leading indication for heart transplant under $18 \mathrm{yr}$ of age (Arola et al. 1997; Nugent et al. 2003; Lipshultz et al. 2003). Cardiomyopathy is often a fatal disease, with 10-yr transplantfree survival rates of $29 \%-94 \%$, depending on type, etiology, and age at presentation (Cox et al. 2006; Wilkinson et al. 2010). Restrictive cardiomyopathy is the rarest form of cardiomyopathy in children, comprising only $3 \%$ of all forms of cardiomyopathy in children, and has the poorest survival with 20\% transplant-free survival at $5 \mathrm{yr}$ (Fenton et al. 2006; Webber et al. 2008). The etiology of restrictive cardiomyopathy (RCM) in most children is not known and therefore is considered idiopathic, although sarcomeric mutations have been reported in some individuals. Autosomal-dominant mutations found in several genes coding for sarcomeric proteins have been associated with RCM, including TNNI3 (MIM: 191044; RCM1, MIM: 115210), TNNT2 (MIM: 191045; RCM3, MIM: 612422), MYPN (MIM: 608517; RCM4, MIM: 615248), ACTC1 (MIM: 102540), and TNN (MIM: 188840) (Kaski et al. 2008; Purevjav et al. 2012; Peled et al. 2014; Starr et al. 2015). Syndromic association of RCM has also been described in some patients with mutations in SMAD4 (MIM: 600993), which lead to Myhre syndrome (MYHRS, MIM: 139210), an autosomal-dominant connective tissue disorder (Starr et al. 2015).

An association between RCM and congenital heart disease (CHD) is not well described, but septal defects in particular have been reported in a few individuals (Yang et al. 2010). Sequencing panels have been the standard of care to date for detecting mutations in genes associated with cardiomyopathy (Hershberger et al. 2009b). Mutation detection rates vary between $20 \%$ and $60 \%$ depending on cardiomyopathy phenotype (Hershberger et al. 2009a), although detection rates in RCM have not been reported. The utilization of copynumber variation (CNV) analysis has identified a number of CNVs associated with an increased risk of CHD (Soemedi et al. 2012; Glidewell et al. 2015). Further, exome sequencing has been used with increasing frequency to identify rare, Mendelian disorders associated with both RCM and CHD (Zaidi et al. 2013; Peled et al. 2014), yet the majority of patients remain without a diagnosis.

CNVs involving 2q13 are enriched in cohorts of patients with intellectual disability (ID), developmental delay (DD) (Cooper et al. 2011), and schizophrenia (Costain et al. 2013). To date, 21 patients with a 2q13 microdeletion have been reported, and a heterogeneous phenotype has started to emerge (Table 1). The recurrent $2 q 13$ microdeletion syndrome is characterized by unique facial dysmorphisms, ID/DD, and microcephaly. CHD has also been reported in patients with the $2 q 13$ microdeletion, although the frequency and severity of cardiac disease is quite varied. Recurrent CNVs involving $2 q 13$ have been reported and commonly include the genes BUB1, ACOXL, BCL2L11, ANAPC1, MERTK, TMEM87B, FBLN7, ZC3H8, and ZC3H6 (Fig. 1). Haploinsufficiency of FBLN7 and TMEM87B in developing zebrafish embryos was associated with cardiac defects suggesting a critical role for these genes in the recurrent 2q13 microdeletion syndrome (Russell et al. 2014).

We describe a patient who presented with dysmorphic features, microcephaly, short stature, metaphyseal dysplasia, ID, and CHD. Chromosomal microarray analysis (CMA) identified a 1.7-Mb loss at 2q13 (Chr 2:111,406,838-113,102,594; hg19), which was determined to be maternally inherited by parental fluorescence in situ hybridization (FISH). The patient's cardiac findings included an atrial septal defect and restrictive cardiomyopathy (RCM). Although many of his phenotypic features were consistent with previous reports of the recurrent $2 q 13$ microdeletion syndrome, whole-exome sequencing (WES) was performed because of inheritance of the CNV from an unaffected parent and to further investigate the etiology of his RCM. WES revealed a paternally inherited, missense variant in TMEM87B. This potentially deleterious variant combined with the loss of the other copy 
Table 1. Patients reported with recurrent $2 q 13$ microdeletion syndrome

\begin{tabular}{|c|c|c|c|c|}
\hline Reference $^{a}$ & $\begin{array}{l}\text { Genomic breakpoints } \\
\text { (GRCh37/hg19) }^{\mathrm{b}}\end{array}$ & $\begin{array}{l}\text { Size } \\
(\mathrm{Mb})\end{array}$ & Inh & Phenotype \\
\hline Bisgaard et al. 2007 & Chr 2:111,439,229-113,060,032 & 1.62 & Mat & Dysmorphism, FTT, Sz, hypotonia, DD, Resp insuff \\
\hline Rudd et al. 2009 (5) & Chr 2:111,442,130-113,065,779 & 1.62 & Unk & Dysmorphism, CHD \\
\hline Rudd et al. 2009 (6) & Chr 2:111,442,130-113,065,779 & 1.62 & Pat & Dysmorphism, speech delay, GERD \\
\hline Rudd et al. 2009 (7) & Chr 2:111,442,130-113,065,779 & 1.62 & Unk & $\begin{array}{l}\text { Dysmorphism, CHD, hypotonia, microcephaly, stiff } \\
\text { joints }\end{array}$ \\
\hline Yu et al. 2012 (6) & Chr 2:111,426,338-113,046,185 & 1.62 & Mat & Microcephaly, hydrocephalus, DD, ID, hypoglycemia \\
\hline Yu et al. 2012 (7) & Chr 2:111,426,338-113,046,185 & 1.62 & Unk & Macrocephaly, scoliosis, DD, ID, pyloric stenosis \\
\hline Yu et al. 2012 (8) & Chr 2:111,399,243-113,008,023 & 1.61 & Unk & NA \\
\hline Yu et al. 2012 (9) & Chr 2:111,335,143-112,746,937 & 1.45 & De novo & NA \\
\hline Yu et al. 2012 (10) & Chr 2:111,399,243-113,007,823 & 1.61 & Unk & NA \\
\hline Yu et al. 2012 (11) & Chr 2:111,442,130-113,065,779 & 1.62 & Pat & Dysmorphism, DD, broad feet, short toes \\
\hline Yu et al. 2012 (12) & Chr 2:111,442,130-113,065,779 & 1.62 & Unk & CHD, Sz, sleep apnea \\
\hline Yu et al. 2012 (13) & Chr 2:111,442,130-113,065,779 & 1.62 & Unk & $\begin{array}{l}\text { Dysmorphism, microcephaly, CHD, hypotonia, } \\
\text { microphallus, inguinal hernia, agenesis corpus } \\
\text { callosum, apnea }\end{array}$ \\
\hline Yu et al. 2012 (14) & NA & NA & Mat & Dysmorphism, DD, Sz, hypoglycemia, resp insuff \\
\hline Yu et al. 2012 (15) & Chr 2:111,616,529-112,995,529 & 1.38 & Unk & ASD \\
\hline Costain et al. 2013 & Chr 2:111,388,632-113,115,993 & 1.73 & Unk & Schizophrenia \\
\hline Ma et al. 2014 & Chr 2:111,832,461-122,620,022 & 10.79 & De novo & $\begin{array}{l}\text { Dysmorphism, short stature, scoliosis, DD, } \\
\text { hypothyroidism, Mullerian agenesis }\end{array}$ \\
\hline Hladilkova et al. 2015 (1) & Chr 2:111,690,367-113,494,060 & 1.8 & Mat & Dysmorphism (mild), microcephaly, DD \\
\hline Hladilkova et al. 2015 (2) & Chr 2:111,690,367-113,477,596 & 1.79 & Unk & Dysmorphism (mild), ASD, PDD-NOS, DD (mild) \\
\hline Riley et al. 2015 (9) & Chr 2:111,442,176-113,065,741 & 1.62 & Pat & Dysmorphism, microcephaly, short stature, CHD \\
\hline Riley et al. 2015 (10) & Chr 2:111,442,131-113,065,779 & 1.62 & Pat & $\begin{array}{l}\text { Dysmorphism, microcephaly, CHD, DD, speech delay, } \\
\text { hypotonia, recurrent bronchitis }\end{array}$ \\
\hline Riley et al. 2015 (11) & Chr 2:111,675,789-113,378,322 & 1.70 & De novo & Macrocephaly, Sz, hypospadias, sleep apnea \\
\hline This report & Chr 2:111,406,838-113,102,594 & 1.70 & Mat & Dysmorphism, microcephaly, short stature, CHD, DD \\
\hline
\end{tabular}

ADHD, attention deficit hyperactivity disorder; ASD, autism spectrum disorder; CHD, congenital heart disease; DD, developmental delay; FTT, failure to thrive; GERD, gastroesophageal reflux disease; ID, intellectual disability; Inh, inheritance; Mat, maternal; NA, not available; Pat, paternal; PDD-NOS, pervasive developmental delay not otherwise specified; Resp insuff, respiratory insufficiency; RP, retinitis pigmentosa; Sz, seizure disorder; Unk, unknown.

${ }^{a}$ The numbers in parentheses indicate the case number in the publication.

${ }^{b}$ Genomic breakpoints converted to GRCh37/hg19 using LiftOver functionality in the UCSC Genome Browser.

of TMEM87B due to the maternally inherited $2 q 13$ microdeletion suggests a recessive condition in our patient.

\section{RESULTS}

\section{Clinical Presentation}

The patient was the result of a full-term delivery complicated by maternal preeclampsia in the third trimester. At birth, he had no respiratory effort and thus required supplementation oxygen, and he was noted to be hypotonic. At 2 mo of age he presented with a respiratory syncytial virus (RSV) infection, tracheomalacia, and bronchiolitis. During that period, hospitalization and an echocardiogram were performed that noted a moderate-sized atrial septal defect (Fig. 2A). Over his first year of life he developed progressive enlargement of his right 


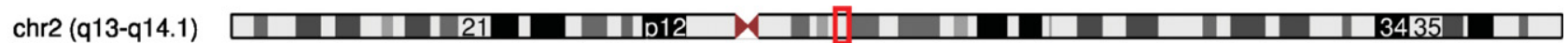

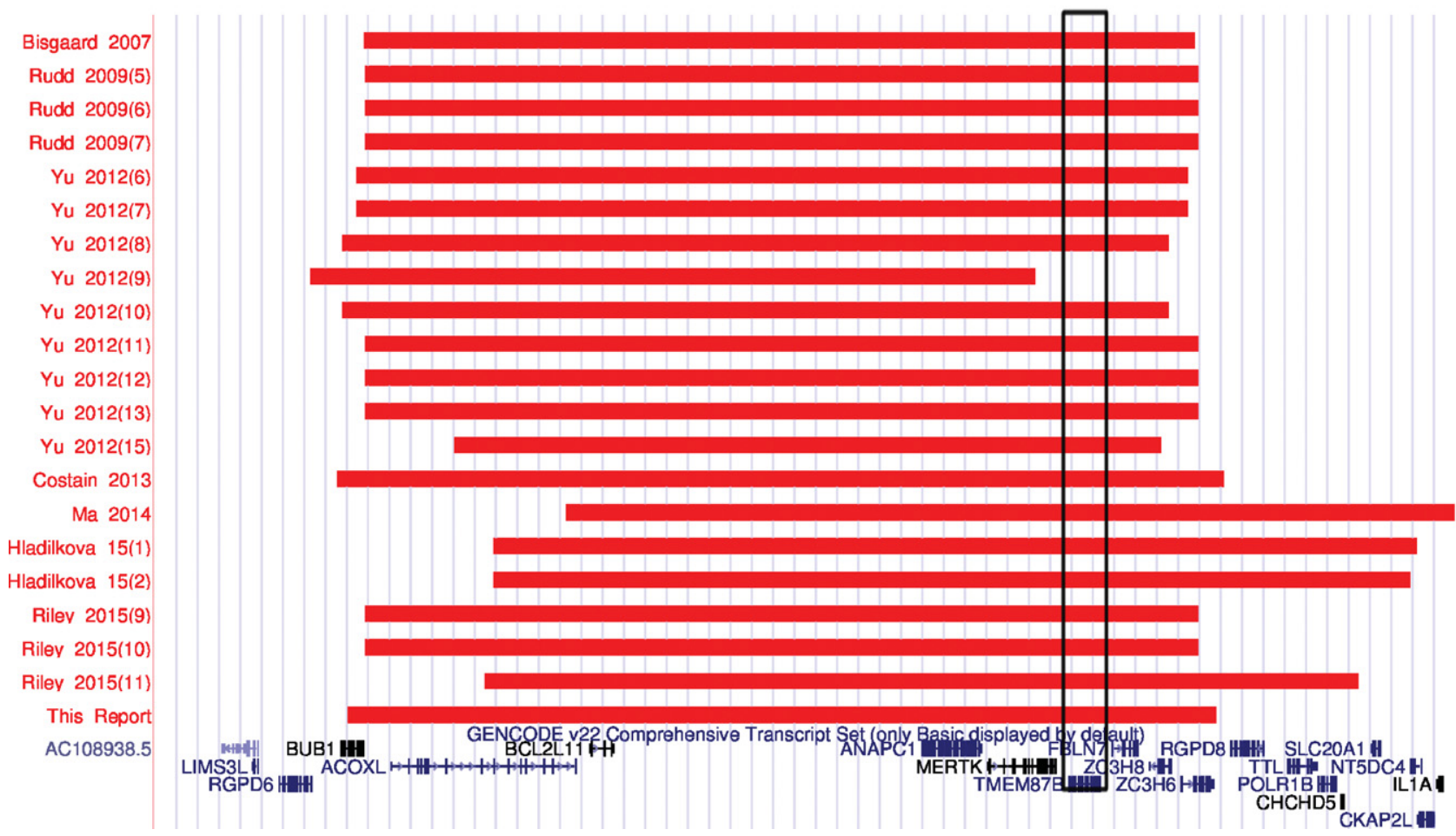

Figure 1. A recurrent 2q13 microdeletion involving TMEM87B. Graphical representation of Chromosome $2 q 13$ microdeletions in 20 previously reported cases and the current report. Minimum CNV sizes are represented by red bars and RefSeq genes (blue) are indicated at the bottom. Each individual case is reported by first author, year of publication, and case number in the publication. A recurrent $2 q 13$ microdeletion is noted despite a heterogeneous phenotype among the reported cases. The majority of $2 q 13$ microdeletions involve TMEM87B (rectangular box). Deletions are based on the UCSC Genome Browser build GRCh37/hg19.

and left atrium with bidirectional flow across his atrial septal defect and increased right ventricular systolic pressures, suggestive of poor compliance of his ventricular myocardium (restrictive physiology). At $15 \mathrm{mo}$, a diagnostic heart catheterization demonstrated elevated end-diastolic pressures of both ventricles, whereas a cardiac MRI showed that the pericardium was normal. This constellation of findings was diagnostic of RCM (Fig. 2B).

His clinical course is otherwise remarkable for mild dysmorphic features including a prominent nasal root, hypertelorism, mildly dysplastic pinnae, and brachydactyly of his hands and feet (Fig. 3A). He has significant short stature (5 SD below the mean) and microcephaly (5 SD below the mean). His skeletal survey noted rizomelic shortening, mild lumbar posterior vertebral body scalloping, and metaphysical irregularity in bilateral femurs (Fig. 3B). He is currently 7 years old and in an age-appropriate academic grade, although he had difficulty with speech and behavioral concerns, including oppositional and aggressive behaviors.

\section{Recurrent 2q13 Microdeletion}

A loss in copy number in the proximal arm of Chromosome 2 was identified by high-resolution CMA in the patient and was later confirmed to be maternally inherited by parental FISH analysis. In the patient, the 2q13 microdeletion (Chr 2:111,406,838-113,102,594; hg19) 


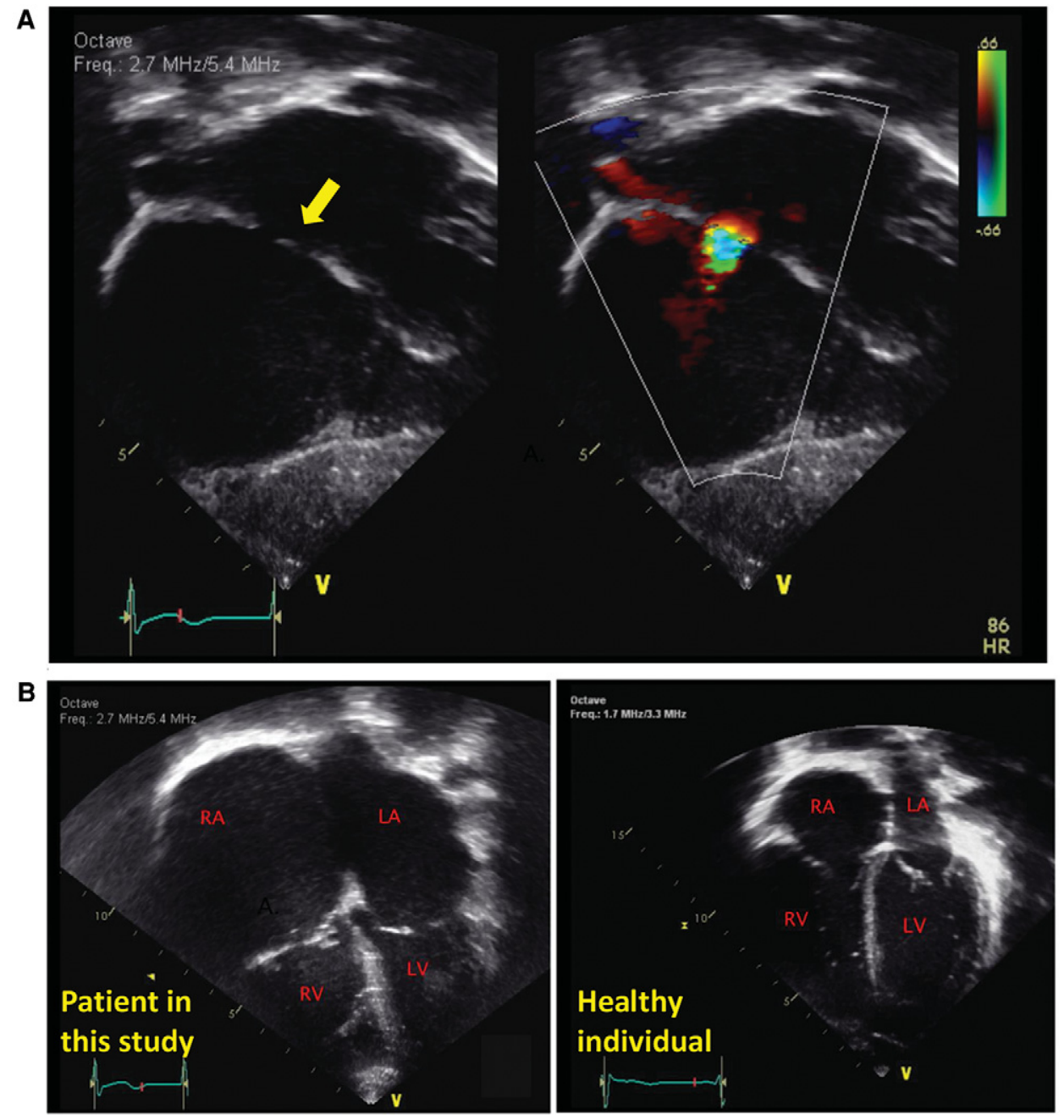

Figure 2. Cardiac findings in a patient with a hemizygous TMEM87B mutation and $2 q 13$ microdeletion. (A) At $2 \mathrm{mo}$, a moderate atrial septal defect (arrow) was noted in this patient. (B) At 8 mo, a significant enlargement of his right atrium was noted with bidirectional flow suggestive of restriction of the right ventricle myocardium. At $15 \mathrm{mo}$, his diagnostic heart catheterization demonstrated restrictive physiology consistent with a restrictive cardiomyopathy. Echocardiogram from a healthy individual is shown on the right. RA, right atrium; LA, left atrium; RV, right ventricle; LV, left ventricle.

spanned $\sim 1.7 \mathrm{Mb}$ and had similar boundaries as other cases with the recurrent $2 \mathrm{q} 13$ microdeletion syndrome (Table 1). The deletion region included several genes: BUB1, ACOXL, BCL2L11, ANAPC1, MERTK, TMEM87B, FBLN7, ZC3H8, and ZC3H6 (Fig. 1). There were two genes that were previously linked to human diseases: Mutations in BUB1 were noted to be a risk factor and associated with colorectal cancer (CRC, MIM: 114500), and mutations in MERTK were found in patients with a form of retinitis pigmentosa (RP38, MIM: 613862). The patient's family history was not suggestive of an increased risk for colorectal cancer or retinitis pigmentosa, and the patient did not exhibit clinical symptoms of these disorders at the time of the evaluation.

The patient had many of the features reported in previous cases with the recurrent $2 q 13$ microdeletion syndrome as shown in Table 1 (Bisgaard et al. 2007; Rudd et al. 2009; Yu et al. 2012; Costain et al. 2013; Ma et al. 2014; Hladilkova et al. 2015; Riley et al. 2015). However, 
A
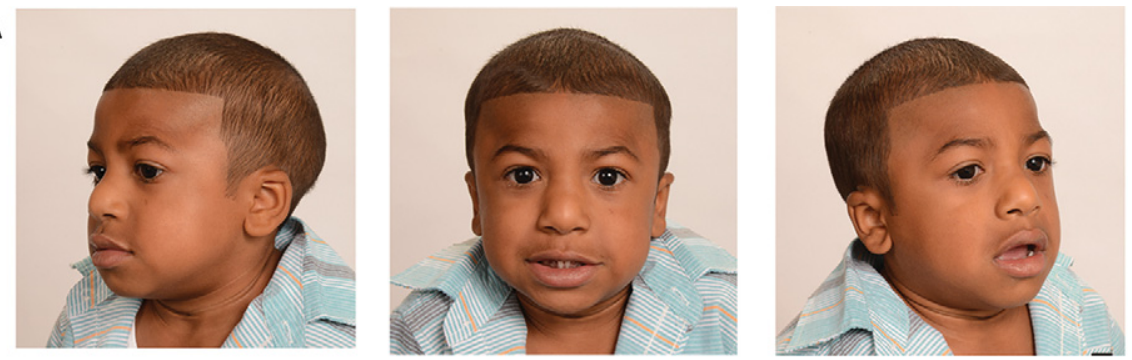

B
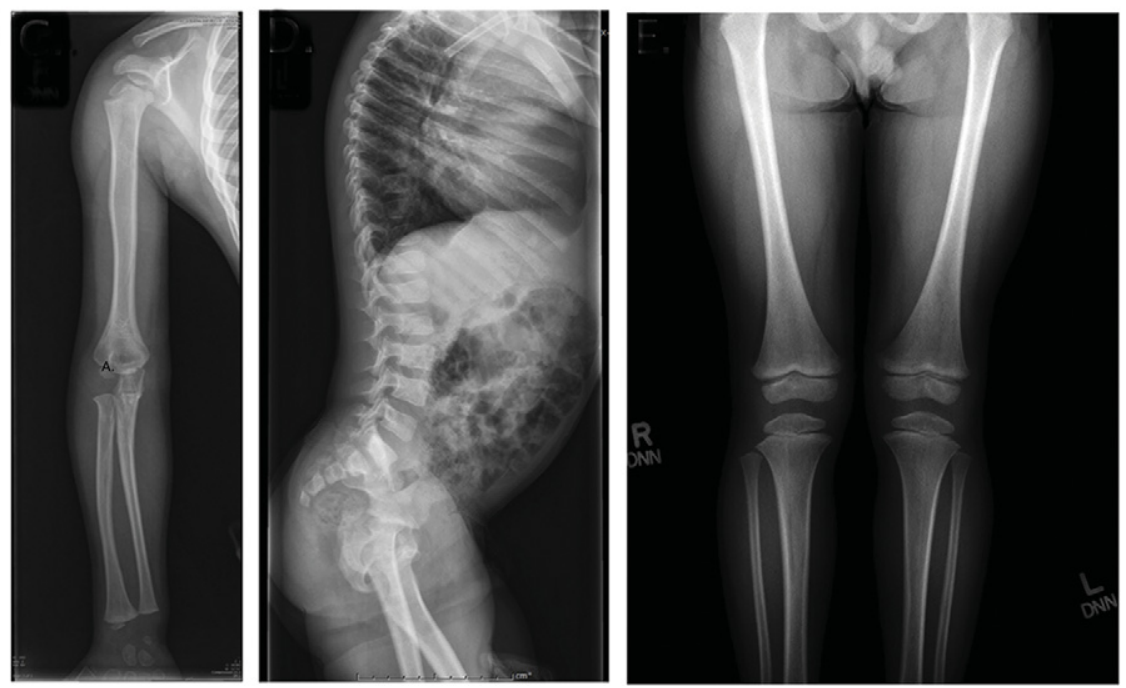

Figure 3. Other clinical findings of the patient in this study. (A) The patient presented with dysmorphic features reported in other patients with recurrent $2 q 13$ microdeletion syndrome including microcephaly, prominent nasal root, hypertelorism, and mildly dysplastic pinnae. (B) The patient also had unique skeletal findings, including rizomelic shortening, mild lumbar posterior vertebral body scalloping, and metaphyseal irregularity in bilateral femurs.

the inheritance of the microdeletion from an unaffected mother combined with the low incidence $(10 \%)$ and milder forms of cardiac defects in previously reported cases made the clinical significance of the CMA results unclear.

\section{Whole-Exome Sequencing}

WES analysis was performed on the patient and his unaffected parents to obtain more than $70 \times$ coverage of targeted exons in each sample (Table 2). A large number of variants

Table 2. Summary of next-generation sequencing (NGS)

\begin{tabular}{lccc}
\hline NGS statistics & Proband & Mother & Father \\
\hline Raw reads & $72,853,546$ & $66,822,418$ & $92,453,366$ \\
Reads mapped to GRCh37/hg19 & $69,824,226$ & $65,763,116$ & $91,052,868$ \\
Reads without duplicates & $60,567,126$ & $57,777,382$ & $81,818,034$ \\
Reads mapped to coding exons & $36,095,699$ & $33,572,650$ & $48,445,183$ \\
Average coverage of coding exons & $76.89 \times$ & $71.62 \times$ & $103.45 \times$ \\
\hline
\end{tabular}


Table 3. Summary of exome variants and test of inheritance models

\begin{tabular}{|c|c|c|c|c|c|}
\hline & Proband & & & & \\
\hline Total variants & 114,576 & & & & \\
\hline Coding variants & 23,569 & & & & \\
\hline $\begin{array}{l}\text { Nonsynonymous, splice-site, } \\
\text { indel variants }\end{array}$ & 11,636 & & & & \\
\hline \multirow[t]{2}{*}{ Rare variants } & 1,952 & & & & \\
\hline & & $\begin{array}{l}\text { Dominant } \\
\text { model }\end{array}$ & \multicolumn{3}{|c|}{ Recessive models } \\
\hline \multirow[t]{3}{*}{ Test of inheritance model } & & De novo & Compound heterozygous & Homozygous & X-linked hemizygous \\
\hline & $\begin{array}{c}\text { Candidate } \\
\text { genes }\end{array}$ & 1 (ETV3L) & $\begin{array}{l}8 \text { (APOB, CAST, LRRIQ4, } \\
\text { SCN3A, EPHA10, ACAN, } \\
\text { KIAA1683, AGBL1) }\end{array}$ & $\begin{array}{l}2(\text { AHNAK, } \\
\left.\text { TMEM87B }{ }^{\mathrm{a}}\right)\end{array}$ & $\begin{array}{l}4 \text { (DLG3, GLRA4, } \\
\text { RBMXL3, BRS3) }\end{array}$ \\
\hline & $\begin{array}{l}\text { Top } \\
\text { candidates }\end{array}$ & 1 (ETV3L) & 2 (APOB, LRRIQ4) & $1\left({\left.\text { TMEM } 87 B^{\mathrm{a}}\right)}^{2}\right.$ & $2(R B M X L 3, B R S 3)$ \\
\hline
\end{tabular}

${ }^{a}$ This variant was originally identified as a homozygous variant. However, after further investigation, it was shown to be a paternally inherited hemizygous missense variant, which appeared to be homozygous because of a maternally inherited 2q13 microdeletion including TMEM87B.

$(114,576)$ were detected in the patient after applying appropriate quality measures (Table 3). Our downstream analyses were focused on nonsynonymous coding variants, coding indels (insertions/deletions) of $<50 \mathrm{bp}$ and variants affecting splice sites as they are more likely to have a functional impact on the gene product and are hence more likely to be pathogenic $(11,636$ variants). We first filtered out common variants with minor allele frequency (MAF) of $>1 \%$ in dbSNP (Database for Short Genetic Variations) and 1000 Genomes data. This resulted in the identification of 1952 rare variants in the patient that were considered for further analysis. Parental WES data were used to detect the pathogenic variant under various inheritance models including dominant (de novo mutations) and recessive (compound heterozygous, homozygous, and X-linked hemizygous mutations) models. This resulted in identification of 15 candidate genes (Table 3; details of variants are provided in Supplemental Table 1). These candidates underwent further evaluation according to the criteria described in the Methods section and resulted in only six genes remaining in our top priority list. They are ETV3L, APOB, LRRIQ4, TMEM87B, RBMXL3, and BRS3 (Table 3). $A P O B$ was the only gene previously known to be associated with human diseases such as hypercholesterolemia (MIM: 144010) and hypo- $\beta$-lipoproteinemia (MIM: 615558), but these were unrelated to our patient's condition.

Interestingly, one of the top candidate genes is TMEM87B, which notably is one of the genes located within the recurrent $2 q 13$ microdeletion. The variant found in TMEM87B (NM_032824.2:c.1366A>G; Chr 2:112856265/hg19) was at first identified as a homozygous mutation according to our bioinformatics pipeline (Table 3). However, after further investigation, it was confirmed that it was a paternally inherited hemizygous variant unmasked by maternally inherited 2q13 microdeletion (Fig. 4A). Variant c.1366A>G found in TMEM87B is extremely rare and also has very high conservation scores across several algorithms (Table 4). This variant is very likely to be pathogenic according to multiple mutation prediction algorithms that we used (Table 4). Change in nucleotide from adenine to guanine at position 1366 subsequently causes p.Asn456Asp missense change in the protein sequence. Amino acid Asn456 is evolutionarily conserved across multiple species (Fig. 4B), and its location (at the end of last transmembrane domain) suggested that it could have a functional impact on the protein (Fig. 4C). Further, we did not find any instances of individuals who were homozygous for this mutation in any publicly available database, including Exome Variant 
COLD SPRING HARBOR Molecular Case Studies
A patient with $2 q 13$ deletion and TMEM87B variation
A

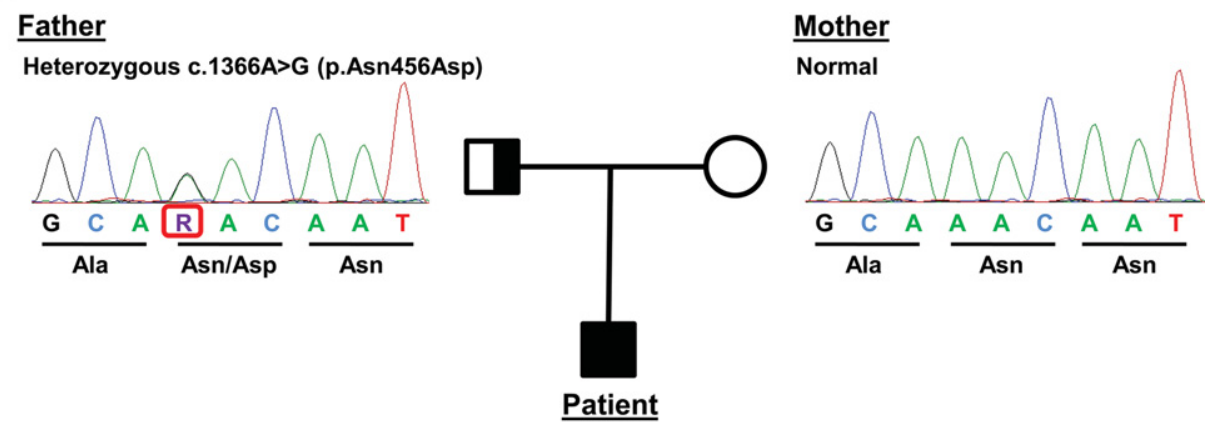

Hemizygous c.1366A>G (p.Asn456Asp)

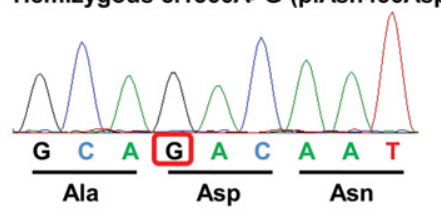

B
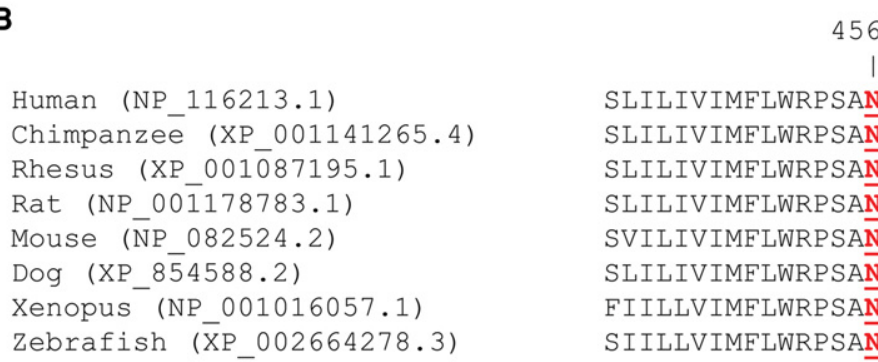

SLILIVIMFLWRPSANNQRYAFMPLIDDSDD SLILIVIMFLWRPSAN̄NQRYAFMPLIDDSDD SLILIVIMFLWRPSANNNQRYAFMPLIDDSDD SLILIVIMFLWRPSANNNQRYAFMPLIDDSDD SVILIVIMFLWRPSAN̄NQRYAFMPLIDDSDD SLILIVIMFLWRPSANNQRYAFMPLIDDSDD FIILLVIMFLWRPSAN̄NQRYAFTPLIDDSDD SI ILLVIMFLWRPSAN̄NQRYAFTPLIDDSDD

C

c. $1366 A>G$ $\uparrow$

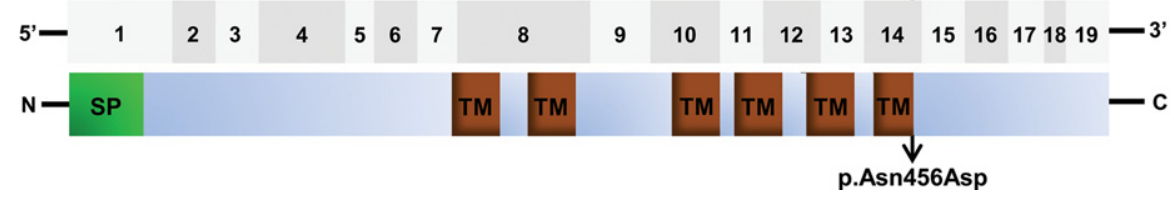

Figure 4. TMEM87B variants in the patient. (A) Partial chromatograms showing Sanger sequencing results of TMEM87B in the patient and his parents. The patient has a paternally inherited, hemizygous missense variant c.1366A>G (p.Asn456Asp). (B) Comparative analysis of TMEM87B from multiple species demonstrates that Asn456 (highlighted in red) is evolutionarily conserved. Protein sequences were obtained from the National Center for Biotechnology Information (NCBI) Protein database. (C) (Top) The 19 coding exons of TMEM87B are shown as gray boxes. (Bottom) The protein domains of TMEM87B, including signal peptide (SP) and six transmembrane domains (TMs) (annotated by Universal Protein Resource, UniProt), are shown. The location of the variant identified in the patients is indicated by arrows.

Server (EVS) or Exome Aggregation Consortium (ExAC). Additionally, we did not observe any individuals who were homozygous or compound heterozygous for loss-of-function (LOF) variants in TMEM87B in these databases, as well as in a large Icelandic population (Sulem et al. 2015).

\section{TMEM87B Mutation Screening}

To evaluate the role of TMEM87B in RCM, samples from 14 patients with isolated RCM were analyzed for mutations in TMEM87B by Sanger sequencing. All patients had a clinical history 


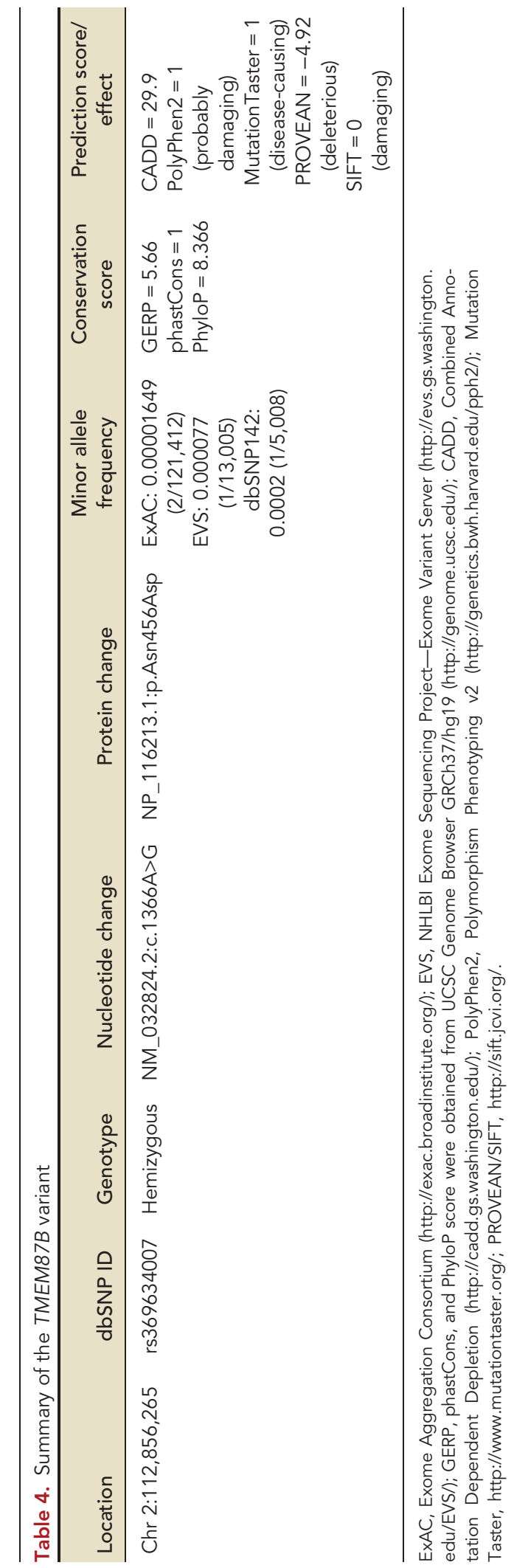


COLD SPRING HARBOR Molecular Case Studies
A patient with $2 q 13$ deletion and TMEM87B variation

of RCM and subsequently received a cardiac transplant. Sanger sequencing of all 19 coding exons and exon-intron boundaries of TMEM87B did not identify potential pathogenic mutations. No other patients with potential TMEM87B mutations were identified.

\section{DISCUSSION}

CNVs are an increasingly recognized cause of disease and have been identified in $\sim 10 \%-$ $20 \%$ of individuals referred for clinical testing (Shaw-Smith et al. 2004; Wagenstaller et al. 2007). Parental testing is often utilized to aid in the interpretation of novel CNVs (Lee et al. 2007), although incomplete penetrance of disease-causing CNVs can result in the inaccurate interpretation that an inherited CNV is benign (Girirajan and Eichler 2010; Rosenfeld et al. 2013). There is a significant level of incomplete penetrance and variable expressivity associated with the recurrent $2 q 13$ microdeletion syndrome. This is highlighted by the reports of a significant number of parents who carry the same $2 q 13$ microdeletion as their affected child but are phenotypically normal. Further, the initial reports of the recurrent $2 q 13$ microdeletion syndrome concluded that the CNV was a benign variant because of the presence of the deletion in asymptomatic family members (Bisgaard et al. 2007; Rudd et al. 2009). The majority of $2 q 13$ microdeletions appear to be inherited, as only three $2 q 13$ microdeletions were noted to be de novo in the previously reported cases (Table 1).

Furthermore, the clinical symptoms of the recurrent $2 q 13$ microdeletion syndrome are quite variable, including two patients with isolated autism spectrum disorder and schizophrenia, respectively (Yu et al. 2012; Costain et al. 2013). It has been suggested that phenotypic variability observed in genetic disorders may be the result of additional genetic factors such as additional CNVs that impact a synergist pathway or a single-nucleotide variant in a related gene (Girirajan et al. 2012). Recently whole-exome sequencing combined with CMA revealed that hemizygous mutations in SNAP29, a gene located within the 22q11.2 microdeletion region, contribute to an extreme phenotype of patients with $22 \mathrm{q} 11.2$ microdeletion syndrome (McDonald-McGinn et al. 2013). These patients not only had common features of 22q11.2 microdeletion syndrome (22q11.2DS, MIM: 188400/MIM: 192430), including cardiac abnormalities, palatal anomalies, and immunodeficiency, they also had additional features of cerebral dysgenesis, neuropathy, ichthyosis, and palmoplantar keratoderma (CEDNIK, MIM: 609528), a recessive condition resulting from unmasking mutations in SNAP29. This finding adds to the increasing list of such cases and underscores the need to consider a second mutation in the nondeleted allele of a gene within the microdeletion region in patients with atypical clinical findings.

Our patient had a maternally inherited 2q13 microdeletion detected by CMA. However, it was suspected that the $2 q 13$ microdeletion was not sufficient to explain all the clinical findings in our patient because of a rather extreme phenotype when compared with other patients with the recurrent 2 q13 microdeletion syndrome. Although our patient's dysmorphic features and intellectual disability were consistent with the majority of cases of the recurrent $2 q 13$ microdeletion syndrome, he also has significant short stature, which was observed in only two previous patients (<3rd percentile for age) (Ma et al. 2014; Riley et al. 2015). Further, our patient also has significant microcephaly and skeletal findings suggestive of a skeletal dysplasia. Microcephaly has been reported in $30 \%$ of the previous case reports, although no patients have such remarkable microcephaly or the associated skeletal findings noted in our patient (Rudd et al. 2009; Yu et al. 2012; Hladilkova et al. 2015; Riley et al. 2015). The $2 q 13$ CNV was first associated with an increased risk of CHD (Soemedi et al. 2012), and one-third of patients with the $2 q 13$ microdeletion thus far have been reported to have $\mathrm{CHD}$. The severity of CHD reported is also quite varied, ranging from atrial septal defects or ventricular septal defects to complex CHD including heterotaxy, aortic coarctation, 
COLD SPRING HARBOR Molecular Case Studies
A patient with $2 q 13$ deletion and TMEM87B variation hypoplastic aortic arch, and hypoplastic left ventricle (Riley et al. 2015). Our patient was noted to have an atrial septal defect, but also RCM, which was not previously reported.

Morpholino-based knockdown of TMEM87B expression resulted in cardiac anomalies in developing zebrafish embryos, suggesting that TMEM87B plays a critical role in cardiac development, which may further explain the observed cardiac phenotype in patients with the recurrent 2q13 microdeletion syndrome (Russell et al. 2014). Our finding of mutations in both alleles of TMEM87B, in an individual with significant cardiac defects, further supports the role of TMEM87B in the cardiac phenotype observed in patients with the recurrent 2 q13 microdeletion syndrome. Thus, it is possible that the phenotypic variability observed in patients with recurrent 2 q13 microdeletion syndrome may be due to additional single-nucleotide variants on the nondeleted allele of one or more genes within this interval. We suggest that in patients with the recurrent $2 q 13$ microdeletion syndrome and extreme phenotypes, the nondeleted copy of genes with the $2 q 13$ microdeletion interval, including TMEM87B, be analyzed for a "second-hit" mutation.

Notably, our patient was identified to have RCM. As opposed to other forms of cardiomyopathy, the majority of cases of RCM do not have a well-defined genetic etiology (SenChowdhry et al. 2010; Lopes and Elliott 2014). Thus, the identification of a novel gene, like TMEM87B, associated with RCM will be useful in improving the content of existing, commercial gene panels used for mutation screening in patients with cardiomyopathy. Our sequence analysis of TMEM87B in 14 other children with RCM of unknown etiology did not identify any mutations, suggesting that TMEM87B is likely to be associated with a rare form of autosomal-recessive RCM. It is tempting to speculate that RCM in association with CHD is more likely to have a genetic etiology with an autosomal-recessive mode of inheritance. Thus, the role of TMEM87B in cardiac development requires further study given the findings in this report, and the fact that others have reported septal defects in combination with RCM (Yang et al. 2010).

By combining CMA and WES, we were able to potentially uncover an autosomal-recessive disorder characterized by a severe cardiac phenotype and caused by hemizygous mutations in TMEM87B. This case emphasizes the importance of interrogating both CNV and genomic sequencing to fully interpret genomic testing results. The interpretation of CMA or WES in isolation was initially inconclusive. In a significant number of patients who undergo CMA and WES testing, a diagnosis remains elusive. It remains to be evaluated whether CMA and WES testing in isolation results in false-negative interpretation of genomic testing.

\section{METHODS}

\section{DNA Extraction}

The patient and his parents were enrolled into a research protocol (COMRIB no. 07-0386) approved by the institutional review board at the University of Colorado School of Medicine. High-quality, unamplified, and unfragmented genomic DNA (A260/A280 $\geq 1.8$ and $A 260 / A 230 \geq 1.9$ ) was extracted from whole blood obtained from the patient and his parents using the Puregene Blood Kit from QIAGEN.

\section{Chromosomal Microarray Analysis}

High-resolution chromosomal microarray analysis (CMA Oligo V8.0) was performed by Baylor College of Medicine (BCM) Medical Genetics Laboratories. The only reported abnormality was a single copy loss of a region on $2 q 13$. This deletion was originally identified in human genome assembly hg18 as Chr 2:111,123,310-112,819,065 and later converted to hg19 as Chr 2:111,406,838-113,102,594 using LiftOver functionality in the UCSC Genome 
COLD SPRING HARBOR Molecular Case Studies
A patient with $2 q 13$ deletion and TMEM87B variation

Browser. $2 q 13$ microdeletion in the patient was determined to be maternally inherited by parental FISH.

\section{WES and Data Analysis}

WES was performed on the patient and his parents using Agilent SureSelectXT Human All Exon V5 followed by sequencing on an Illumina HiSeq 2000. Details of data analysis were similar to the procedure as previously described (Yu et al. 2013). Approximately 50 million, 100-bp, paired-end reads $(>70 \times)$ were obtained and mapped to the reference human genome (GRCh37/hg19) using the Burrows-Wheeler aligner (BWA) (Li and Durbin 2009a) (summarized in Table 2). Variants were determined by the utilities in the SAMtools (Li et al. 2009b) and further annotated using SeattleSeq. Filtering and test of the inheritance model were done using tools in Galaxy (Goecks et al. 2010). Variants were filtered against dbSNP build 137, 1000 Genomes (November 23, 2010 release version), and Exome Aggregation Consortium ExAC browser (version 0.3). Rare variants were identified as variant with minor allele frequency (MAF) of $<1 \%$ using dbSNP137. The sequence data from the family was then used to test for causal variants under different inheritance models, including de novo mutation in a dominant model and compound heterozygous, homozygous, and X-linked hemizygous mutations in recessive models. Candidate genes were further evaluated based on different criteria in each model. In the dominant model, variants found in any database (dbSNP, 1000 Genomes, ExAC) were removed from the top candidacy list. In the recessive model, we also removed autosomal variants that had homozygotes found in the databases (or variants on $\mathrm{Chr} \mathrm{X}$ or $\mathrm{Chr} \mathrm{Y}$ with hemizygotes in databases) from the top candidacy list.

\section{Sanger Sequencing Verification}

Validation of the mutation was done by PCR amplification followed by Sanger sequencing using genomic DNA from the patient and his parents $(50 \mathrm{ng})$, and forward primer of 5'-TGGAAGACTTACTGGTTGGAAAG-3' and reverse primer of 5'-CCCAGTTCAGTCATTC GCTATC-3'. The PCR conditions were as follows: Promega GoTaq Hot Start kit with 1× Master Mix and $400 \mathrm{~nm}$ of each primer. PCR began with an initial cycle at $95^{\circ} \mathrm{C}$ for $3 \mathrm{~min}$, followed by 30 cycles of $94^{\circ} \mathrm{C}$ for $30 \mathrm{sec}, 60^{\circ} \mathrm{C}$ for $30 \mathrm{sec}$ and $72^{\circ} \mathrm{C}$ for $1 \mathrm{~min}$, finishing with extension at $72^{\circ} \mathrm{C}$ for $5 \mathrm{~min}$. Amplified PCR products were sequenced using the PCR primers as sequencing primers on an $\mathrm{ABI}$ PRISM 3730xI at a commercial sequencing facility. Variations detected in TMEM87B were named using cDNA accession number NM_032824.2.

\section{Additional Samples}

Patients were enrolled into a research protocol (COMRIB no. 04-0891) approved by the institutional review board at the University of Colorado School of Medicine; explants were retrieved in the operating room and flash frozen in liquid nitrogen for storage at $-80^{\circ} \mathrm{C}$. Genomic DNA was extracted from heart tissue using DNeasy Blood and Tissue kit from QIAGEN using the manufacture's recommendations for tissue extraction. The conditions of PCR and sequencing were as described above. Primer pairs were designed to amplify and sequence a total of 19 exons in TMEM87B (NM_032824.2). The list of primer sequences is provided in the Supplemental Table 2.

\section{ADDITIONAL INFORMATION}

\section{Ethics Statement}

The index patient and his parents were enrolled into a research protocol (COMIRB no. 07-0386) approved by the Institutional Review Board at the University of Colorado School 
$8^{\infty} \mathrm{C}$ C O L D S PR ING HARBOR Molecular Case Studies
A patient with $2 q 13$ deletion and TMEM87B variation
Competing Interest Statement

The authors have declared no competing interest.

Received December 18, 2015; accepted in revised form February 3, 2016. of Medicine. Written informed consent was obtained from the patient and his parents. Fourteen additional patients with restrictive cardiomyopathy were enrolled into a research protocol (COMIRB no. 04-0891) approved by the Institutional Review Board at the University of Colorado School of Medicine, and written consent was obtained from the patients.

\section{Data Deposition and Access}

WES data is not publicly available because patient consent could not be obtained. The TMEM87B variant and $2 q 13$ microdeletion found in the patient of this study have been deposited in ClinVar (http://www.ncbi.nlm.nih.gov/clinvar/) under accession numbers SCV000255630 and SCV000255631, respectively.

\section{Acknowledgments}

We are very grateful to the patients and their families for participating in this study.

\section{Author Contributions}

H.-C.Y., C.R.C., and T.H.S. designed the study. C.R.C. and K.C.C. contributed to patient recruitment. C.R.C., E.R.E., J.L.C., K.C.C., and S.D.M. collected and interpreted clinical data. E.A.G. extracted patient's genomic DNA. H.-C.Y. analyzed whole-exome sequencing data. H.-C.Y., C.R.C., E.R.E., and T.H.S. contributed to interpretation of sequencing data. H.-C.Y., E.A.G., and K.C.C. performed TMEM87B screening. H.-C.Y., C.R.C., and T.H.S. contributed to the writing of the manuscript. All authors contributed to editing and reviewing the final draft.

\section{Funding}

This work was supported in part by the National Institutes of Health (GM081519 to T.H.S).

\section{REFERENCES}

Arola A, Jokinen E, Ruuskanen O, Saraste M, Pesonen E, Kuusela AL, Tikanoja T, Paavilainen T, Simell O. 1997. Epidemiology of idiopathic cardiomyopathies in children and adolescents. A nationwide study in Finland. Am J Epidemiol 46: 385-393.

Bisgaard AM, Kirchhoff M, Nielsen JE, Brandt C, Hove H, Jepsen B, Jensen T, Ullmann R, Skovby F. 2007. Transmitted cytogenetic abnormalities in patients with mental retardation: pathogenic or normal variants? Eur J Med Genet 50: 243-255.

Cooper GM, Coe BP, Girirajan S, Rosenfeld JA, Vu TH, Baker C, Williams C, Stalker H, Hamid R, Hannig V, et al. 2011. A copy number variation morbidity map of developmental delay. Nat Genet 43: 838-846.

Costain G, Lionel AC, Merico D, Forsythe P, Russell K, Lowther C, Yuen T, Husted J, Stavropoulos DJ, Speevak M, et al. 2013. Pathogenic rare copy number variants in community-based schizophrenia suggest a potential role for clinical microarrays. Hum Mol Genet 22: 4485-4501.

Cox GF, Sleeper LA, Lowe AM, Towbin JA, Colan SD, Orav EJ, Lurie PR, Messere JE, Wilkinson JD, Lipshultz SE. 2006. Factors associated with establishing a causal diagnosis for children with cardiomyopathy. Pediatrics 118: 1519-1531.

Fenton MJ, Chubb H, McMahon AM, Rees P, Elliott MJ, Burch M. 2006. Heart and heart-lung transplantation for idiopathic restrictive cardiomyopathy in children. Heart 92: 85-89.

Girirajan S, Eichler EE. 2010. Phenotypic variability and genetic susceptibility to genomic disorders. Hum Mol Genet 19: R176-R187.

Girirajan S, Rosenfeld JA, Coe BP, Parikh S, Friedman N, Goldstein A, Filipink RA, McConnell JS, Angle B, Meschino WS, et al. 2012. Phenotypic heterogeneity of genomic disorders and rare copy-number variants. N Engl J Med 367: 1321-1331. 
Glidewell SC, Miyamoto SD, Grossfeld PD, Clouthier DE, Coldren CD, Stearman RS, Geraci MW. 2015. Transcriptional impact of rare and private copy number variants in hypoplastic left heart syndrome. Clin Transl Sci 8: 682-689.

Goecks J, Nekrutenko A, Taylor J. 2010. Galaxy: a comprehensive approach for supporting accessible, reproducible, and transparent computational research in the life sciences. Genome Biol 11: R86.

Hershberger RE, Cowan J, Morales A, Siegfried JD. 2009a. Progress with genetic cardiomyopathies: screening, counseling, and testing in dilated, hypertrophic, and arrhythmogenic right ventricular dysplasia/cardiomyopathy. Circ Heart Fail 2: 253-261.

Hershberger RE, Lindenfeld J, Mestroni L, Seidman CE, Taylor MR, Towbin JA; Heart Failure Society of America. 2009b. Genetic evaluation of cardiomyopathy - a Heart Failure Society of America practice guideline. J Card Fail 15: 83-97.

Hladilkova E, Barøy T, Fannemel M, Vallova V, Misceo D, Bryn V, Slamova I, Prasilova S, Kuglik P, Frengen E. 2015. A recurrent deletion on chromosome $2 q 13$ is associated with developmental delay and mild facial dysmorphisms. Mol Cytogenet 8: 57.

Kaski JP, Syrris P, Burch M, Tomé-Esteban MT, Fenton M, Christiansen M, Andersen PS, Sebire N, Ashworth M, Deanfield JE, et al. 2008. Idiopathic restrictive cardiomyopathy in children is caused by mutations in cardiac sarcomere protein genes. Heart 94: 1478-1484.

Lee C, lafrate AJ, Brothman AR. 2007. Copy number variations and clinical cytogenetic diagnosis of constitutional disorders. Nat Genet 39: S48-S54.

$\mathrm{Li} \mathrm{H}$, Durbin R. 2009a. Fast and accurate short read alignment with Burrows-Wheeler transform. Bioinformatics 25: $1754-1760$.

Li H, Handsaker B, Wysoker A, Fennell T, Ruan J, Homer N, Marth G, Abecasis G, Durbin R. 2009b. The Sequence Alignment/Map format and SAMtools. Bioinformatics 25: 2078-2079.

Lipshultz SE, Sleeper LA, Towbin JA, Lowe AM, Orav EJ, Cox GF, Lurie PR, McCoy KL, McDonald MA, Messere JE, et al. 2003. The incidence of pediatric cardiomyopathy in two regions of the United States. N Engl J Med 348: 1647-1655.

Lopes LR, Elliott PM. 2014. A straightforward guide to the sarcomeric basis of cardiomyopathies. Heart 100: 1916-1923.

Ma D, Marion R, Punjabi NP, Pereira E, Samanich J, Agarwal C, Li J, Huang CK, Ramesh KH, Cannizzaro LA, et al. 2014. A de novo 10.79 Mb interstitial deletion at 2q13q14.2 involving PAX8 causing hypothyroidism and Mullerian agenesis: a novel case report and literature review. Mol Cytogenet 7: 85.

McDonald-McGinn DM, Fahiminiya S, Revil T, Nowakowska BA, Suhl J, Bailey A, Mlynarski E, Lynch DR, Yan AC, Bilaniuk LT, et al. 2013. Hemizygous mutations in SNAP29 unmask autosomal recessive conditions and contribute to atypical findings in patients with 22q11.2DS. J Med Genet 50: 80-90.

Nugent AW, Daubeney PE, Chondros P, Carlin JB, Cheung M, Wilkinson LC, Davis AM, Kahler SG, Chow CW, Wilkinson JL, et al. 2003. The epidemiology of childhood cardiomyopathy in Australia. N Engl J Med 348: 1639-1646.

Peled Y, Gramlich M, Yoskovitz G, Feinberg MS, Afek A, Polak-Charcon S, Pras E, Sela BA, Konen E, Weissbrod O, et al. 2014. Titin mutation in familial restrictive cardiomyopathy. Int J Cardiol 171: 24-30.

Purevjav E, Arimura T, Augustin S, Huby AC, Takagi K, Nunoda S, Kearney DL, Taylor MD, Terasaki F, Bos JM, et al. 2012. Molecular basis for clinical heterogeneity in inherited cardiomyopathies due to myopalladin mutations. Hum Mol Genet 21: 2039-2053.

Riley KN, Catalano LM, Bernat JA, Adams SD, Martin DM, Lalani SR, Patel A, Burnside RD, Innis JW, Rudd MK. 2015. Recurrent deletions and duplications of chromosome $2 q 11.2$ and $2 q 13$ are associated with variable outcomes. Am J Med Genet A 167: 2664-2673.

Rosenfeld JA, Coe BP, Eichler EE, Cuckle H, Shaffer LG. 2013. Estimates of penetrance for recurrent pathogenic copy-number variations. Genet Med 15: 478-481.

Rudd MK, Keene J, Bunke B, Kaminsky EB, Adam MP, Mulle JG, Ledbetter DH, Martin CL. 2009. Segmental duplications mediate novel, clinically relevant chromosome rearrangements. Hum Mol Genet 18: 2957-2962.

Russell MW, Raeker MO, Geisler SB, Thomas PE, Simmons TA, Bernat JA, Thorsson T, Innis JW. 2014. Functional analysis of candidate genes in 2q13 deletion syndrome implicates FBLN7 and TMEM87B deficiency in congenital heart defects and FBLN7 in craniofacial malformations. Hum Mol Genet 23: 4272-4284.

Sen-Chowdhry S, Syrris P, McKenna WJ. 2010. Genetics of restrictive cardiomyopathy. Heart Fail Clin 6: 179-186.

Shaw-Smith C, Redon R, Rickman L, Rio M, Willatt L, Fiegler H, Firth H, Sanlaville D, Winter R, Colleaux L, et al. 2004. Microarray based comparative genomic hybridisation (array-CGH) detects submicroscopic chromosomal deletions and duplications in patients with learning disability/mental retardation and dysmorphic features. J Med Genet 41: 241-248. 
Soemedi R, Wilson IJ, Bentham J, Darlay R, Töpf A, Zelenika D, Cosgrove C, Setchfield K, Thornborough C, Granados-Riveron J, et al. 2012. Contribution of global rare copy-number variants to the risk of sporadic congenital heart disease. Am J Hum Genet 91: 489-501.

Starr LJ, Grange DK, Delaney JW, Yetman AT, Hammel JM, Sanmann JN, Perry DA, Schaefer GB, Olney AH. 2015. Myhre syndrome: clinical features and restrictive cardiopulmonary complications. Am J Med Genet $A$ 167: 2893-2901.

Sulem P, Helgason H, Oddson A, Stefansson H, Gudjonsson SA, Zink F, Hjartarson E, Sigurdsson GT, Jonasdottir A, Jonasdottir A, et al. 2015. Identification of a large set of rare complete human knockouts. Nat Genet 47: 448-452.

Wagenstaller J, Spranger S, Lorenz-Depiereux B, Kazmierczak B, Nathrath M, Wahl D, Heye B, Glaser D, Liebscher V, Meitinger T, et al. 2007. Copy-number variations measured by single-nucleotide-polymorphism oligonucleotide arrays in patients with mental retardation. Am J Hum Genet 81: 768-779.

Webber S, Lipshultz SE, Sleeper LA, Wilkinson JD, Addonizio LJ, Canter CE, Everitt MD, Kantor P, Lamour JM, Lu M, et al. 2008. Phenotypic heterogeneity and outcomes of restrictive cardiomyopathy in childhood-a report from the NHLBI pediatric cardiomyopathy registry. Circulation 118: S1055.

Wilkinson JD, Landy DC, Colan SD, Towbin JA, Sleeper LA, Orav EJ, Cox GF, Canter CE, Hsu DT, Webber SA, et al. 2010. The pediatric cardiomyopathy registry and heart failure: key results from the first 15 years. Heart Fail Clin 6: 401-413.

Yang SW, Hitz MP, Andelfinger G. 2010. Ventricular septal defect and restrictive cardiomyopathy in a paediatric TNNI3 mutation carrier. Cardiol Young 20: 574-576.

Yu HE, Hawash K, Picker J, Stoler J, Urion D, Wu BL, Shen Y. 2012. A recurrent 1.71 Mb genomic imbalance at $2 q 13$ increases the risk of developmental delay and dysmorphism. Clin Genet 81: 257-264.

Yu HC, Sloan JL, Scharer G, Brebner A, Quintana AM, Achilly NP, Manoli I, Coughlin CR II, Geiger EA, Schneck U, et al. 2013. An X-linked cobalamin disorder caused by mutations in transcriptional coregulator HCFC1. Am J Hum Genet 93: 506-514.

Zaidi S, Choi M, Wakimoto H, Ma L, Jiang J, Overton JD, Romano-Adesman A, Bjornson RD, Breitbart RE, Brown KK, et al. 2013. De novo mutations in histone-modifying genes in congenital heart disease. Nature 498: 220-223. 


\section{COLD SPRING HARBOR Molecular Case Studies}

\section{Discovery of a potentially deleterious variant in TMEM87B in a patient with a hemizygous 2 q13 microdeletion suggests a recessive condition characterized by congenital heart disease and restrictive cardiomyopathy}

Hung-Chun Yu, Curtis R. Coughlin, Elizabeth A. Geiger, et al.

Cold Spring Harb Mol Case Stud 2016, 2: a000844 originally published online February 8, 2016 Access the most recent version at doi: $10.1101 /$ mcs.a000844

Supplementary Material

References

License

Email Alerting Service
http://molecularcasestudies.cshlp.org/content/suppl/2016/02/08/mcs.a000844.D C1

This article cites 40 articles, 6 of which can be accessed free at: http://molecularcasestudies.cshlp.org/content/2/3/a000844.full.html\#ref-list-1

This article is distributed under the terms of the Creative Commons Attribution-NonCommercial License, which permits reuse and redistribution, except for commercial purposes, provided that the original author and source are credited.

Receive free email alerts when new articles cite this article - sign up in the box at the top right corner of the article or click here. 